\title{
PAUL BERNAYS ET LA RÉNOVATION DES FONDEMENTS PHILOSOPHIQUES DES MATHÉMATIQUES
}

\author{
Gerhard HEINZMANN
}

\begin{abstract}
RÉSUMÉ : L'histoire des fondements des mathématiques du $\mathrm{XX}^{\mathrm{e}}$ siècle montre qu'il nous faut réviser la signification des notions philosophiques traditionnelles comme "évidence », « existence », « expérience » ou « rationalité ». On expose comment le logicien Paul Bernays, familier des conceptions de la philosophie de Jacob Friedrich Fries et de Léonard Nelson, donne aux résultats techniques une interprétation philosophique dont il s'inspire de plus en plus - à partir du milieu du siècle - de la «philosophie ouverte » de Ferdinand Gonseth. Bien avant Thomas Kuhn, Bernays conçoit la révision envisagée non pas simplement comme une question de vérité ou de fausseté, mais comme un problème exigeant l'introduction d'un nouveau système conceptuel. Cependant, l'affaiblissement du concept de vérité est pour lui une conséquence épistémologique provenant de la théorie de la connaissance adoptée et non une conséquence méthodologique issue d'un changement de paradigme et donc d'une incommensurabilité.
\end{abstract}

Mots-CLÉS : Bernays, Gonseth, intuition, structures schématiques, « expérience mentale », complémentarité.

ABSTRACT : The history of the foundations of mathematics in the XX ${ }^{\text {th }}$ century leads to a revision of the meaning of traditional philosophical notions such as " evidence ", " existence 》, " experience " or "rationality». This paper focuses on the influence of Ferdinand Gonseth's "open philosophy » on Paul Bernays' philosophical interpretations of the technical results in the foundations. Familiar with the ideas of Jacob Friedrich Fries and Léonard Nelson, the logician Bernays turns at the middle of the century more and more towards Gonseth's ideas. Quite before Thomas Kuhn, Bernays considers the revisions under consideration not simply as a problem of truth and falsehood, but as a task requiring the introduction of a new conceptual system. Indeed, in opposition to Kuhn, according to Bernays, the necessary change is less the consequence from a change of paradigm and methodological incommensurability as a epistemological necessity.

KEYWORDS : Bernays, Gonseth, intuition, schematic structures, mental experience, complementarity.

Revue de synthèse : 5érie, année 2005/2, p. 317-329. 
Zusammenfassung : Die Geschichte der Grundlagen der Mathematik im 20. Jahrhundert belegt die Notwendigkeit einer Revision der Bedeutung von traditionellen philosophischen Begriffen wie « Evidenz », « Existenz », «Erfahrung » oder « Rationalität ». Der Gegenstand dieses Artikels ist der Einfluss von Ferdinand Gonseths «Offener Philosophie » auf die philosophische Interpretation von Paul Bernays' technischen Resultaten in den Grundlagen. Vertraut mit den Ideen von Jacob Friedrich Fries und Léonard Nelson, wendet sich der Logiker Bernays zur Mitte des Jahrhunderts mehr und mehr den Ideen Gonseths zu. Schon vor Thomas Kuhn betrachtet Bernays dabei die anstehende Revision nicht einfach als ein Problem von Wahrheit oder Falschheit, sondern als die Aufgabe der Einführung eines neuen konzeptuellen Systems. Der Grund ist aber hierfür weniger ein Paradigmenwechsel oder methodologische Inkommensurabilität als erkenntnistheoretische Notwendigkeit.

STICHWÖRTER: Bernays, Gonseth, Anschauung, schematische Strukturen, Geistesexperiment, Komplementarität.

Gerhard Heinzmann, né en 1950, a fait ses études de mathématiques et de philosophie à Fribourgen-Brisgau, Heidelberg, Sarrebruck et Paris VII. Assistant de recherche au Collège de France (19811983), Hochschulassistent à l'université de la Sarre, il devient en 1990 maître de conférences, puis professeur à l'université Nancy 2. Directeur du «Laboratoire de philosophie et d'histoire des sciences - Archives Henri-Poincaré» (UMR 7117 du CNRS), ses recherches portent sur la philosophie de la logique et des mathématiques et tout particulièrement sur l'œuvre de Poincaré. Mais ses domaines d'intérêts concernent également les aspects systématiques de la philosophie de la connaissance, la philosophie grecque, l'histoire des sciences, l'empirisme logique et la philosophie du langage ; il a publié de nombreux travaux sur les œuvres de Charles Sanders Peirce, Henri Poincaré, Ferdinand Gonseth et Jean Cavaillès que l'on pourra consulter sur le site Internet : <http ://www.univ-nancy2.fr/poincare >.

Adresse : Laboratoire de philosophie et d'histoire des sciences - Archives Henri-Poincaré, Université de Nancy 2, 23 bd Albert-Irer, F-54015 Nancy Cedex.

Courrier électronique : Gerhard.Heinzmann@univ-nancy2.fr 
La naissance, autour de 1900, de la logique symbolique, de la théorie des ensembles et du nouveau rôle que l'on attribue à la méthode axiomatique est accompagnée d'un aspect unificateur qui se manifeste déjà dans le choix des titres d'ouvrages. En effet, depuis le Formulaire de mathématiques ${ }^{1}$ de Giuseppe Peano, les fondements des mathématiques remplacent les fondements de la géométrie et de l'arithmétique et restent pendant tout le siècle un titre courant. Commençant avec The Principles of mathematics de Bertrand Russell ${ }^{2}$ et terminant avec The Principles of mathematics revisited ${ }^{3}$ de Jaakko Hintikka, on trouve un grand nombre d'ouvrages sur les fondements, les principes, les éléments ou la philosophie des mathématiques ${ }^{4}$. La majeure partie de ces études concerne la théorie des ensembles et la logique, domaines qui sont abordés selon trois différents « soucis fondationnels » auxquels s'ajoute un intérêt technique :

a) on les analyse en poursuivant un intérêt d'unification et de clarification. Ainsi on insiste sur la généralité, le nombre minimal de termes primitifs et d'axiomes, la clarté du langage ;

b) on les analyse en se plaçant dans un contexte épistémologique. La consistance, l'existence, la relation entre objets mathématiques et objets physiques, et la question qu'est-ce qu'une démonstration? jouent alors un rôle primordial ;

c) on les analyse en visant un intérêt historique ;

d) on les analyse dans un intérêt technique.

Du point de vue de la logique classique, les deux premières approches ont été poursuivies à la fois par les philosophes et les mathématiciens. La grande majorité des questions abordées au XIX ${ }^{\mathrm{e}}$ siècle tirent leur origine de la discussion sur les monstres et sur la formalisation des mathématiques. Il s'agit en fait d'une critique du rationalisme classique qui prétend que l'on dispose d'une aperception claire et distincte des concepts dans l'évidence rationnelle ${ }^{5}$.

Si cette critique visait ce que les Anglais appellent un «framework for the working mathematicien », la flexibilité technique de l'axiomatisation ensembliste donnerait un résultat satisfaisant et Bernays y aurait contribué d'une manière substantielle. Par contre, si cette critique concernait le fondement philosophique, le résultat semblerait décevant : la théorie axiomatisée des ensembles ( $1^{\mathrm{er}}$ ordre) est déductivement incomplète, inévitablement non standard et nous ne pouvons avoir une idée claire de ce que son modèle intentionné caractérise. En d'autres termes, nous ne pouvons nous fier à la théorie formelle sans renoncer à notre intuition d'un contenu conceptuel.

On se demande alors si la traductibilité dans un langage logique et ensembliste classique est le seul critère de rigueur et de justification, si l'on veut maintenir l'intégralité scientifique. Une réponse pourrait être « Mathematics without foundations ${ }^{6}$ ». L'existence

1. PEANO, 1895-1908.

2. RUSSELL, 1903.

3. HinTIKKa, 1996.

4. Pour plus de détails concernant les thèses soutenues dans cette introduction, voir HEINZMANN, 2004.

5. Vuillemin, 1979.

6. Titre d'un article devenu célèbre de Putnam, 1967. 
de propositions formellement indécidables (dans un système arithmétique donné) ou les problèmes non résolus par les axiomes standard (en théorie des ensembles) n'ont pas empêché le développement d'une science viable et, en fait, puissante. En conséquence, c'est l'idée même de fondements des mathématiques qui pourrait être suspecte. Les mathématiques pourraient alors être comprises à partir de leur seule pratique. Certes, on se rappelle la leçon des Lumières : ce qui caractérise le philosophe est l'abandon d'un système normatif en faveur d'un portrait du comportement. « Le philosophe, dit Diderot, est celui qui sait s'arrêter juste ${ }^{7}$. » Tout en reconnaissant cet enseignement, ne

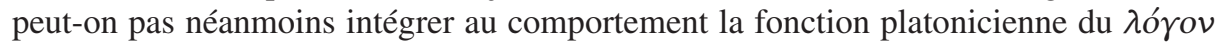
$\delta \iota \delta o ́ v \alpha \iota ?$

En fait, la critique de l'évidence rationnelle - au début du siècle un leitmotiv bien justifié pour l'entreprise des fondements - ne doit pas revenir, en mathématiques, à son élimination pure et simple en faveur du raisonnement logique ; sinon, l'évidence analytique serait non suspecte tout comme l'évidence en métamathématique. La conscience que les mathématiques formalisées ne peuvent être privées d'un élément intuitif pourrait bien être la devise de Bernays, pourtant l'un des créateurs dans le domaine de la théorie axiomatique des ensembles.

Né en 1888 à Londres, fils d'un homme d'affaires qui s'installera bientôt à Berlin, Bernays commence ses études de mathématiques, de philosophie et de physique théorique dans la capitale de la Prusse où il suit entre autres les cours de Issai Schur, Edmund Landau, Georg Ferdinand Frobenius, Carl Stumpf, Ernst Cassirer et Max Planck. Après deux ans et demi, il passe à Göttingen et y assiste aux cours de David Hilbert, Edmund Landau, Hermann Weyl, Félix Klein, Max Born, Woldemar Voigt et Léonard Nelson qui était alors le représentant principal de l'école néofriesienne. D'ailleurs, la première publication de Bernays, imprimée dans les Abhandlungen der Friesschen Schule, a comme titre Das Moralprinzip bei Sidgwick und bei Kant. En 1912, Bernays soutient sa thèse de doctorat sur un sujet de la théorie analytique des nombres sous la direction de Landau. La même année encore, il obtient son habilitation à Zurich où Ernst Zermelo était depuis 1910 professeur titulaire - ce dernier travail porte sur la théorie des fonctions. Il devient alors Privatdozent. En contact avec George Polya, Albert Einstein et Hermann Weyl, il rencontre en automne 1917 David Hilbert à Zurich lorsque celuici donne sa célèbre conférence Axiomatisches Denken. Hilbert l'invite alors à retourner à Göttingen et à collaborer comme son assistant à ses recherches sur les fondements de l'arithmétique, qu'il avait récemment reprises. En 1919, Bernays obtient à Göttingen la venia legendi avec un travail axiomatique sur le calcul propositionnel des Principia mathematica. À partir de 1922, il est außerordentlicher Professor dans cette même ville. Dès 1929, il se consacre à la transformation du système neumanien de la théorie des ensembles, travail qu'il ne publiera qu'à partir de 1937 parce qu'il était mécontent du caractère artificiel de l'axiomatisation impliquée ${ }^{8}$. Après avoir perdu sa venia legendi en 1933 - c'est Weyl qui faisait soutenir à sa place la thèse de Saunders Mac Lane qu'il dirigeait ${ }^{9}-$, il reste encore six mois assistant, à titre privé, de Hilbert avant de retourner comme chargé de cours à Zurich. Il ne parvient qu'en 1939 à obtenir à nouveau sa

7. Diderot, 1986, vol. II, article « Socratique », p. 320.

8. BERnAYs, 1976b, p. XVI.

9. Nous remercions Ralf Kroemer pour ce renseignement. 
venia legendi ${ }^{10}$, perdue en Allemagne, et il restera Privatdozent jusqu'à 1945, année où il devient enfin professeur à l'École polytechnique fédérale (EPF).

Or, depuis 1929, Gonseth est titulaire, à l'EPF, d'une chaire de « Mathématiques en langue française », mais effectue ses recherches en philosophie des sciences. Gonseth, né en 1890, donc cadet de deux ans de Bernays, présenta en 1917 son travail de Privatdozent à l'EPF ${ }^{11}$, au moment où Bernays quitta l'université de Zurich pour Göttingen. Mais, dès la fin des années 1930, sa coopération avec Gonseth sera constante et s'exprime par l'organisation commune de colloques - il s'agit des Entretiens de Zurich ${ }^{12}$-, la fondation de structures académiques - ensemble, avec Karl Dürr et Karl Popper, ils fondent en 1947 la Société internationale de logique et de philosophie des sciences -, la discussion publiée des idées de son collègue à l'occasion des hommages rendus ${ }^{13}$, des publications communes ${ }^{14}$ et surtout l'édition d'une revue - il s'agit évidemment de Dialectica, fondée en 1947 avec Gaston Bachelard. Dès le premier numéro de Dialectica, Bernays parle de «notre école de philosophie ouverte » lorsqu'il essaie de repousser les interprétations qui cherchent à rapprocher trop vite la dialectique de Gonseth de celle de Hegel ${ }^{15}$.

Comment s'explique cette affinité ? D'abord, Bernays peut assurer au philosophe le lien avec la recherche technique actuelle dans les fondements des mathématiques que Gonseth, atteint de cécité, avait perdu. Ensuite, Gonseth peut procurer au mathématicien un cadre philosophique adéquat. Le passage suivant, extrait de la brève biographie de Bernays que l'on trouve dans Sets and classes, montre en effet que la relation entre les deux savants est loin d'être unilatérale :

«Im Philosophischen kam ich in näheren Kontakt mit Ferdinand Gonseth, an dessen philosophischen Seminarien ich mich mehrmals beteiligte. Ich war aufgrund meiner gedanklichen Auseinandersetzungen mit der Kant-Fries-Nelson'schen Philosophie den Ansichten Gonseth's sehr nahe gekommen, und so schloß ich mich seiner philosophischen Schule an ${ }^{16}$. »

10. BERNAYs, 1976b, p. XVI et SPECKER, 1979, p. 381 sq.

11. Voir EMERY, 1985, p. 13.

12. Bernays donne des contributions aux premiers, deuxièmes, troisièmes et quatrièmes entretiens, voir BERNAYS, 1939b, 1948a, 1952a, 1952b et 1953b.

13. Voir les hommages rendus par BERnAYs, 1950, 1960, 1970 et 1977, à l'occasion des 60 $60^{\mathrm{e}} 7 \mathrm{e}^{\mathrm{e}}$ et $80^{\mathrm{e}}$ anniversaires de Gonseth ainsi qu' après sa mort.

14. Voir Bernays, 1939a ou les actes des premiers Entretiens de Zurich, mis au point, selon les propos de Gonseth, « dans une étroite collaboration » avec Bernays.

15. Voir BERnAYs, 1947, p. 308.

16. BERnAYs, 1976b, p. XVI. Mise à part l'utilisation fréquente du vocabulaire philosophique de Gonseth - voir, p. ex., Bernays, 1976a, p. 80, 87, 91, 95, 107, 172 sq. et 176 sqq. -, Bernays a commenté à plusieurs reprises la «philosophie ouverte» de Gonseth d'une manière explicite: en 1948, il donne aux deuxièmes Entretiens de Zurich une conférence intitulée « Grundsätzliches zur "philosophie ouverte" ", voir BERNAYs, 1948a ; en 1952, lors des troisièmes Entretiens de Zurich, il esquisse sa philosophie en six thèses tout en soulignant la proximité méthodique de la «philosophie ouverte », voir BERNAYS, 1952b ; en 1960, il écrit en hommage au 70e anniversaire de Gonseth un article sur les "Charakterzüge der Philosophie Gonseths", voir BernaYs, 1960 ; à l'occasion de son $80^{\mathrm{e}}$ anniversaire, il rédige l'article « Die schematische Korrespondenz und die idealisierten Strukturen », voir BERNAYS, 1970, reproduit dans les Abhandlungen zur Philosophie der Mathematik, voir BERNAYS, 1976a, et, après la mort de Gonseth, il publie finalement dans Dialectica l'article « Überlegungen zu Ferdinand Gonseths Philosophie », voir BERNAYs, 1977. 
Comment doit-on interpréter cette prétendue parenté intellectuelle entre l'école néofriesienne et la pensée de Gonseth ?

Grâce à l'étude de Volker Peckhaus ${ }^{17}$, on connaît bien la campagne entreprise par Hilbert en faveur de la création à Göttingen d'un « Extraordinariat » pour la philosophie systématique des sciences exactes, poste prévu pour Nelson qu'il obtient finalement en 1919. En fait, Nelson constate «bien des analogies entre l'axiomatique moderne (d'Hilbert) et la critique de la raison (de Fries) », car selon Fries :

«[...] la tâche principale de la philosophie des mathématiques [...] est de distinguer ce qui peut être démontré par la seule logique et ce qui est fondé sur l'intuition. Ainsi le but principal est d'obtenir un petit nombre d'axiomes, mais néanmoins suffisant pour obtenir les autres propositions. [...] Le développement successif de l'axiomatique est affiné de telle sorte que l'on peut prouver les limites de la logique en mathématiques en ce sens qu'il est devenu possible de montrer que l'axiome des parallèles n'est pas dérivable des autres axiomes. Cependant, remarque Nelson, la non-contradiction de la négation d'une proposition n'est rien d'autre que le critère kantien d'une proposition synthétique ${ }^{18}$. »

Le passage de la pensée de Bernays d'éléments nelsoniens à des éléments gonsethiens peut être localisé en ce point précis de l'interprétation axiomatique. Cependant, si l'on choisit comme échelle de réflexion une période de dix ans, le passage prend la forme d'une évolution subtile; si l'on choisit une échelle plus courte, on s'aperçoit que Nelson et Gonseth sont pour ainsi dire rejoints par une courbe possédant deux points d'inversion. Car, pour l'axiomatique hilbertienne du début du siècle, les axiomes ne sont pas évidents et ne sont donc plus le résultat d'une connaissance, d'où la nécessité de chercher une démonstration de non-contradiction. Miriam Franchella nous rapporte que Bernays, lorsqu'il discute un article de Nelson, soutient en 1927 contre Nelson justement une position qui refuse aux axiomes tout caractère abstractif ${ }^{19}$. Appelons cette position formaliste. Par contre, il est particulièrement intéressant de rappeler le but que poursuit Bernays dans l'ouvrage A system of axiomatic set theory, publié sous la forme d'une série d'articles entre 1937 et $1954^{20}$, et qui compte parmi les contributions majeures en théorie des ensembles. L'axiomatisation n'est plus entendue au sens formaliste:

«The theory is not set up as a pure formalism, but rather in the usual manner of elementary axiom theory, where we have to deal with propositions which are understood to have a meaning, and where the reference to the domain of facts to be axiomatized is suggested by the names for the kinds of individuals and for the fundamental predicates ${ }^{21}$. 》

En effet, un système axiomatique de la théorie des ensembles ne peut avoir le même statut qu'un système axiomatique pour la théorie des groupes ou des nombres. Dans ces deux cas, il est absolument correct de considérer les modèles de la théorie, tandis

17. Peckhaus, 1990.

18. FRANCHELLA, 1997, p. 18-19 (c'est nous qui traduisons).

19. Franchella, 1997, p. 18-20.

20. BERNAYS, 1976c.

21. BERNAYS, 1976c, p. 1. 
que parler des modèles de la théorie des ensembles, considérée elle-même comme cadre conceptuel des mathématiques, ne fait aucun sens ${ }^{22}$.

Quoi qu'il en soit, après les travaux de Kurt Gödel et de Gerhard Gentzen, Bernays remarque en 1938 :

«Le récent développement de la théorie de la démonstration de Hilbert [...] a mené à une révision du point de vue philosophique [...Celui-ci nous force à] reconnaître qu'il n'est pas possible de tracer une ligne de démarcation nette entre les raisonnements évidents (sûrs) et les raisonnements attaquables (suspects), quoiqu'on puisse délimiter différents points de vue méthodiques ${ }^{23}$. »

Les différents points de vue méthodiques découlent par exemple des travaux de Gentzen qui suggèrent une séparation nette des méthodes arithmétiques proprement dites de celles appartenant à la théorie cantorienne des ordinaux. L'équivalence montrée par Gödel des raisonnements classiques et intuitionnistes dans le domaine de la théorie des nombres entiers montre, d'une part, la consistance de l'arithmétique et permet, d'autre part, d'établir que la méthode de Gentzen (induction jusqu'à $\varepsilon_{0}$ ) constitue un terme intermédiaire entre la méthode « évidente » finitiste et la méthode « attaquable » de l'intuitionnisme. Or, la révision nécessaire des catégories gnoséologiques d' « évident » ou «attaquable», entendues comme alternative entre l'évidence logique (le fini) et l'évidence empirique ou opératoire, n'est pas suffisamment aboutie par l'introduction d'un synthétique a priori comme le prône la solution kantienne ${ }^{24}$. Les anciennes catégories - «évident », « fini »/« attaquable », « infini »- ne sont plus assez spécifiques. C'est dans la reconnaissance de ce fait que se situe la différence entre Nelson et Gonseth par rapport à Bernays. Il faut, pour le dire en termes gonsethiens, passer d'un niveau épistémologique « intuitif » à un niveau épistémologique «théorique ». Ainsi, la démarcation se retrouve dans la distinction entre le domaine arithmétique et celui d'un certain segment non-initial de la théorie des ensembles. La séparation ne s'opère, dira en 1940 Jean Cavaillès, non pas entre le fini et l'infini mais à l'intérieur des ordinaux de la $2^{\mathrm{e}}$ classe $^{25}$, donc à l'intérieur d'un infini conceptualisé.

Bernays a très bien vu (et avant Kuhn) qu'une révision n'est pas simplement une question de vérité ou de fausseté, mais qu'il s'agit d'introduire un nouveau système conceptuel $^{26}$. Cependant, l'affaiblissement du concept de vérité est pour lui une conséquence épistémologique provenant de la théorie de la connaissance adoptée et non une conséquence méthodologique provenant d'un changement de paradigme et donc d'une incommensurabilité. Comme pour Poincaré et Cassirer, les révisions se font dans le même paradigme, c'est-à-dire d'une manière évolutive et non révolutionnaire.

Dans ses thèses exposées au IX ${ }^{\mathrm{e}}$ Congrès international de philosophie, tenu en 1937 à Paris, Bernays recommande encore d'autres révisions. Elles concernent la distinction entre l'intuition arithmétique et l'intuition géométrique, effectuée traditionnellement selon les éléments temporels et spatiaux. Ce critère devrait être abandonné en faveur

22. MÜLLER, éd., 1981, p. 268.

23. BERNAYS, 1938 (c'est nous qui traduisons).

24. BERNAYs, 1939a, p. 87.

25. Cavaillès, 1962, p. 261.

26. BERNAYs, 1948a, p. 275 et 1952a, p. 136. 
d'une distinction entre le discret et le continu ${ }^{27}$. De plus, au lieu de maintenir la distinction kantienne entre analytique et synthétique, distinction dont les difficultés sont aujourd'hui bien connues par les écrits de Quine, il recommande l'introduction de la distinction entre les éléments d'une théorie motivés formellement et « objectuellement» («gegenständlich») ${ }^{28}$.

C'est par le biais de cette dernière distinction que Bernays arrive à lier les principes gonsethiens de révision et de dualité entre théorie et expérience. En s'opposant à l'empirisme logique, par leur conviction qu'il est impossible de saisir le fait brut par une simple description, Gonseth et Bernays ${ }^{29}$ - et avant eux, Poincaré - développent une méthode d'interaction entre les éléments du langage théorétique et observationnel. Comme le fait déjà Popper, Gonseth et Bernays soulignent que la rationalité n'est pas liée à une évidence absolue. Il nous faut adopter une position du juste milieu entre le programme initial de l'empirisme logique et le tournant, initié par le second Wittgenstein, vers une ordinary philosophy of langage.

Dans cette perspective, le principe de dualité est l'expression d'un point de vue antidualiste en théorie de la connaissance car il postule, avant tout, la thèse selon laquelle la spontanéité et la réceptivité sont inséparables. La compréhension est une capacité et non une théorie interprétative s'exerçant sur une donnée factuelle inaliénable. « L'information que la sensation extérieure nous donne des choses n'a pas un caractère d'immédiateté ${ }^{30} »$ :

«[Es liegt nahe], unsere Erfahrungserkenntnis nicht als einen in der Hauptsache rezeptiven Vorgang und auch nicht als ein unmittelbares Schauen, sondern als ein von Sinneseindrücken angeregtes Erzeugnis des Geistes anzusehen, und [damit behauptet man nicht], daß in diesem Geistesprodukt alles Grundsätzliche durch unveränderliche Grundbeschaffenheiten des Geistes bestimmt sei ${ }^{31}$. »

Ainsi, selon Bernays, il nous faut «d'un point de vue de la "philosophie ouverte" une révision par rapport à la délimitation d'éléments rationnels et empiriques ${ }^{32} »$ : dans un corpus systématique, l'élément rationnel doit, certes, être situé au niveau des expressions d'une théorie scientifique, mais il y est à un stade particulièrement développé ; moins développé, l'élément rationnel se trouve néanmoins déjà dans le mythe tel que celui-ci est à considérer, par rapport à certaines intentions, comme vraie doctrine de la réalité. S'il y a bien différence entre mythe et théorie scientifique, elle est cependant graduelle et non de principe, c'est-à-dire qu'il n'y a pas de frontière franche. De cette façon, la juxtaposition des principes de révision et de dualité conduit à une transformation pragmatique de la corrélation entre les termes en question : d'une part, l'empirique (dans sa signification révisée) n'est pas opposé au rationnel en général mais à une systématisation, c'est-à-dire que l'entendement ne se fait plus valoir dans des principes a priori mais par le progrès de la formation des concepts $^{33}$; d'autre part, le rationnel (dans sa

\footnotetext{
27. BERnAYS, 1976a, p. 81.

28. BERNAYS, 1976 a, p. 79.

29. BERnAYs, 1949, p. 46 sq.

30. BERNAYS, 1946, p. 321.

31. BERNAYS, 1937, p. 282.

32. BERNAYS, 1953a, p. 32.

33. BERNAYS, 1937, p. 289.
} 
conception révisée) n'est pas opposé à l'empirique, mais à la capacité de concevoir des impressions de contenu (inhaltliche Eindrücke), c'est-à-dire de concevoir un domaine de données. Il en découle la possibilité pour Bernays de parler de l'expérience, soit-elle geistig (mentale) ${ }^{34}$, par rapport au domaine des mathématiques. L'opposition kantienne entre le concept et l'intuition qui, exprimée en termes logiques, est l'opposition entre le général et le singulier, se trouve remplacée par la distinction entre forme et contenu, conçue dans la tradition néokantienne comme rapport fonctionnel ${ }^{35}$. L'invariance qui fonde l'analogie entre les concepts d' « expérience » et d' « expérience mentale » consiste dans le fait qu'ils sont tous deux conçus en tant que contenus dans un rapport fonctionnel avec leur forme respective. Ainsi, une révision ne consiste plus dans une critique de la sensibilité par l'entendement (comme le pensait Kant), mais c'est le même entendement qui révise d'un point de vue plus étendu la connaissance antérieure ${ }^{36}$.

Par exemple, en logique, il nous faut bien accepter l'exigence méthodique de la cohérence d'une théorie. Pourtant cette cohérence en tant que forme n'est pas à confondre avec la formulation du principe de non-contradiction en tant qu'instantiation puisqu'il pourrait être susceptible d'amendements ${ }^{37}$. La logique paraconsistante donnera bien raison à Bernays. En mathématique, chaque thématisation schématisante d'un certain domaine, c'est-à-dire le passage d'un domaine particulier à sa structure, nous offre évidemment la possibilité de considérer une foule de domaines structurellement identiques, mais différents quant à leurs contenus. À ce stade, l'élément fonctionnel devient visible grâce à la distinction entre axiomatisation schématisante et structurante, une distinction dont la terminologie est reprise par Bernays de l'ouvrage de Gonseth Le Problème du temps ${ }^{38}$. Les deux façons d'axiomatiser se placent à des niveaux d'abstractions différents. L'axiomatisation schématisante se fonde sur un langage dont l'engagement ontologique est supposé connu et par lequel on dispose de noms propres ou d'un domaine de quantification fixe. Les éléments qui le composent sont d'ailleurs bien différents des objets de la nature, puisque le domaine ne contient pas les faits exprimés dans les assertions élémentaires sur ces éléments ${ }^{39}$. Par contre, dans l'axiomatisation structurante, les objets ne sont uniquement connus que comme membres d'une structure, elle-même donnée par une définition explicite grâce à l'indication de ses axiomes. Les modèles non nécessairement isomorphes qui réalisent cette structure - ou, s'ils ne sont pas isomorphes, qui réalisent le genre de la structure - peuvent êtres considérés comme sa signification extérieure, c'est-à-dire comme son contenu « empirique » conçu. S'exprimer de cette manière se trouve justifiée par le double processus d'abstraction obtenu à la suite d'une concaténation des deux axiomatisations. Bien que les structures soient souvent considérées indépendamment de leurs modèles ou uniquement par rapport à leur signification « interne », l'intérêt plus ou moins grand des modèles, c'est-à-dire leur parenté avec d'autres structures abstraites ou concrètes, peut néanmoins influencer le développement des mathématiques. C'est la raison pour laquelle Bernays réfute avec Gonseth l'opposition carnapienne entre langage théorétique, utilisé pour exprimer un fait formel, et langage observationnel,

34. BERNAYs, 1952a, p. 131.

35. CASSIRER, 1990, p. 343.

36. Bernays, 1948a, p. 276.

37. Bernays, 1948a, p. 278.

38. Bernays, 1970, ici 1976a, p. 181 ; GoNSETH, 1964, p. 159.

39. Bernays, 1963, p. 50. 
utilisé pour exprimer un fait concret ou un fait de la nature. La sémantique des deux langages est supposée être interconnectée dès le départ par une correspondance schématique qui peut intervenir sous plusieurs formes, par exemple comme axiomatisation schématisante et structurante. Citons une conférence de Bernays, faite en 1956 :

«What we do by our mathematical theories of branches of nature is to set up a schema, as Mr. Gonseth says or more explicitly: the theories stand to nature in a relation of schematic correspondence. But these schemata have their inner structure and mathematics can be conceived as the general science of these structures ${ }^{40}$. »

Au contraire, un fait par rapport auquel les points de vue de Paul Bernays et de Rudolf Carnap semblent s'accorder est que l'existence, dans la pratique mathématique, se trouve le plus souvent conditionnée par le cadre langagier choisi en vue d'un objectif arrêté ; elle est donc « interne » (Carnap) ou «bezogen » (Bernays). Mais veut-on alors dire avec Carnap ${ }^{41}$ que le choix du cadre est soumis au principe de tolérance, applicable aux formes linguistiques et déterminé par des considérations pratiques de sorte que les assertions existentielles externes sont dépourvues de tout contenu cognitif ? Ou croit-on, au contraire, le libre choix limité par un engagement ontologique ou par l'intuition mathématique ? On pourrait penser qu'il s'agit ici d'une controverse rappelant la dispute entre le formaliste et le réaliste face au théorème d'incomplétude de Gödel : le formaliste montre la transcendance et par conséquent le non-sens des questions sémantiques, le réaliste considère l'incomplétude comme symptôme de la limitation du formalisme ${ }^{42}$ qui serait à élargir, par exemple dans le sens où Gentzen l'a fait. Cependant, une telle alternative est pour ainsi dire neutralisée ou transgressée par l'opinion avancée par Gonseth, puis par Quine (et non l'inverse) : Gonseth, Quine et Bernays sont d'accord sur le fait qu'il n'existe pas, en principe, une frontière précise entre l'acceptation d'une structure langagière et l'acceptation d'une assertion formulée dans ce langage ${ }^{43}$. Nous sommes ici au cœur de la problématique que Bernays attache au concept de correspondance schématique. Si les propositions théoriques à l'intérieur d'un cadre linguistique ne sont pas nettement distinctes des jugements pratiques qui déterminent ce cadre, il nous faut envisager à la fois un processus de construction des objets et de leur description à l'intérieur du cadre formel suggéré par la construction effectuée. En d'autres termes, un processus constructif de schématisation et un processus descriptif de structuration constituent deux horizons d'objectivité qui restent dans une interdépendance appelée, selon une proposition provenant de Cavaillès, « dialectique ${ }^{44}$ » - d'où le choix du nom Dialectica. Bernays préfère à juste titre l'expression « horizon d'objectivité » à la formulation «horizon de réalité » 45 de Gonseth, et le terme «complémentarité » à celui de «dialectique » ${ }^{46}$. D'une part, car il n'est guère convaincant que les mathématiques puissent être réduites au réel quelle que soit la signification que l'on veut lui

40. BERNAYS, 1956, p. 7.

41. CARNAP, 1958.

42. McNaughton, 1957.

43. Carnap, 1958, n. 1, p. 215 ; Heinzmann, 1982, n. 1, p. 122.

44. BERNAYS, 1977, p. 121.

45. BERNAYS, 1977, p. 125 sq.

46. Bernays, 1948b et 1962. 
attribuer : le réel objectif, le réel phénoménal ou la chose concrète ${ }^{47}$. D'autre part, la dialectique envisagée par Bernays n'est pas simplement un mouvement entre deux états qui s'excluent mutuellement, mais elle ressemble plutôt à la complémentarité entre deux aspects méthodiques. La théorie ondulatoire et la théorie corpusculaire, prises dans le contexte de la théorie quantique, servent d'exemple : aucune n'est complètement adéquate, mais là où l'une échoue, l'autre s'applique ${ }^{48}$. C'est cette révision du concept de réel vers un élément objectuel qui constitue le développement essentiel qu'apporte Bernays à la «philosophie ouverte ».

Gerhard HeINZMANN

(novembre 2000). 


\section{LISTE DES RÉFÉRENCES}

BERNAYs (Paul), 1937, « Grundsätzliche Betrachtungen zur Erkenntnistheorie », Abhandlungen der Friesschen Schule, neue Folge 6, Heft 3-4, p. 279-290.

Bernays (P.), 1938, «Résumé », ms, archives Henri-Poincaré, code Ar-Ju, Université de Nancy 2.

BERnAYs (P.), 1939a, « Bemerkungen zur Grundlagenfrage », in Gonseth (Ferdinand), Philosophie mathématique, Paris, Hermann, p. 83-87.

BERNAYs (P.), 1939b, « Sur les questions méthodologiques actuelles de la théorie hilbertienne de la démonstration », in GoNSETH (Ferdinand), éd., Entretiens de Zurich 1938, Zurich, Leemann \& Co., p. 144-152.

BERNAYS (P.), 1946, «Quelques points de vue concernant le problème de l'évidence », Synthese, vol. V, p. 321-329.

Bernays (P.), 1947, « Contradiction et non-contradiction », Dialectica, vol. I, p. 305-309.

BERNAYS (P.), 1948a, « Grundsätzliches zur "philosophie ouverte” », Dialectica, vol. II, p. 275-279.

Bernays (P.), 1948b, « Über die Ausdehnung des Begriffes der Komplementarität auf die Philosophie », Synthese, vol. VII, p. 66-70.

Bernays (P.), 1949, «Die Erneuerung der rationalen Aufgabe», in Beth (Evert W.), Pos (Henry-J.), HollaK (J. H.), eds, Proceedings of the $X^{\text {th }}$ International Congress of philosophy, Amsterdam, North Holland, vol. I, p. 42-50.

BERNAYs (P.), 1950, « Mathematische Existenz und Widerspruchsfreiheit », in ID., Abhandlungen zur Philosophie der Mathematik, Darmstadt, Wissenschaftliche Buchgesellschaft, 1976, p. 92-106.

Bernays (P.), 1952a, « Dritte Gespräche von Zürich », Dialectica, vol. VI, p. 130-136.

Bernays (P.), 1952b, « Gesichtspunkte der Erkenntnistheorie », Dialectica, vol. VI, p. 137-140.

BernaYs (P.), 1953a, « Diskussionsbemerkungen zum Referat von Herrn Husson », Dialectica, vol. VII, p. 32-34.

Bernays (P.), 1953b, « Einleitendes Referat », Dialectica, vol. VII, p. 318-321.

BERNAYs (P.), 1956, «Language, mathematics and knowledge », Lecture given at Princeton University, 25 April 1956, ms, archives Henri-Poincaré, Université de Nancy 2.

Bernays (P.), 1960, « Charakterzüge der Philosophie Gonseths », Dialectica, vol. XIV, p. 151-156.

BERNAYS (P.), 1962, « Über den Unterschied zwischen realistischer und konservativer Tendenz in der heutigen theoretischen Physik », Revue de métaphysique et de morale, t. LXVII, 2, p. 142-146.

Bernays (P.), 1963, «Remarks to "the End of a Phase" », Dialectica, vol. XVI, p. 49-50.

BERNAYs (P.), 1970, «Die schematische Korrespondenz und die idealisierten Strukturen », Dialectica, vol. XXIV, p. 53-66, réimpr. in BERNAYs, 1976a, p. 176-188.

BERnAYs (P.), 1976a, Abhandlungen zur Philsophie der Mathematik, Darmstadt, Wissenschaftliche Buchgesellschaft.

Bernays (P.), 1976b, « Kurze Biographie », in MÜLleR, 1976, p. XIV-XVI.

Bernays (P.), 1976c, A system of axiomatic set theory, in MüLlER, 1976, p. 1-119.

BERNAYs (P.), 1977, « Überlegungen zu Ferdinand Gonseths Philosophie », Dialectica, vol. XXXI, p. 119-128. 
CARnAP (Rudolf), 1958, « Empiricism, semantics and ontology », in ID., Meaning and necessity. A study in semantics and modal logic, Chicago/Londres, University of Chicago Press, p. 205-221.

CASSIRER (Ernst), 1990, Substanzbegriff und Funktionsbegriff, $1^{\text {re }}$ éd. Berlin, 1910, ici Darmstadt, Wissenschaftliche Buchgesellschaft.

CAvaillès (Jean), 1962, «Transfini et continu », in ID., Philosophie mathématique, Paris, Hermann, p. 255-274.

Diderot (Denis), 1986, Encyclopédie ou dictionnaire raisonné des sciences, des arts et des métiers, chronol., introd. et bibliogr. par Alain Pons, Paris, Flammarion, 2 vol.

EMERY (Éric), 1985, Ferdinand Gonseth. Pour une philosophie dialectique ouverte à l'expérience, Lausanne, L'Âge d'Homme.

Franchella (Miriam), 1997, Paul Bernays' epistemological way towards Gonseth's theory, Milan, Cooperativa Universitaria Editrice Milanese.

Gonseth (Ferdinand), 1964, Le Problème du temps, Neuchâtel, Griffon.

HeInZMANN (Gerhard), 1982, Schematisierte Strukturen. Eine Untersuchung über den Idoneismus Ferdinand Gonseths auf dem Hintergrund eines konstruktivistischen Ansatzes, Berne, Haupt.

HeInZMANN (G.), 2004, «Some coloured remarks on the foundations of mathematics in the $\mathrm{XX}^{\text {th }}$ century », in RAhMAN (Shahid), Symons (John), GABbay (Dove) et VAN BENDEGEM (Jean-Paul), éd., Logic, epistemology and the unity of science, Dordrecht/Boston/Londres, Kluwer, p. 41-50.

HintikKa (Jaakko), 1996, The Principles of mathematics revisited, Cambridge, Cambridge University Press.

McNaughton (Robert), 1957, «Conceptual Schemes in set theory », The Philosophical Review, 61, p. 66-80.

MüLLER (Gert-Heinz), 1976, Sets and classes, Amsterdam/Oxford, North Holland.

MÜLLER (G.-H.), éd., 1981, « Framing mathematics », Epistemologia, IV, 1, p. 253-286.

Peano (Giuseppe), 1895-1908, Formulaire de mathématiques, Turin/Paris, Bocca/Carré et Naud, 5 vol.

PeCKHAus (Volkert), 1990, Hilbertprogramm und Kritische Philosophie, Göttingen, Vandenhoeck et Ruprecht.

Putnam (Hilary), 1967, "Mathematics without foundations », The Journal of Philosophy, vol. LXIV, réimpr. in ID., Philosophical papers, $2^{\mathrm{e}}$ éd., Cambridge, Cambridge University Press, 1993, vol. I, p. 43-59.

Russell (Bertrand), 1903, The Principles of mathematics, 2e éd. Londres, 1979, ici Londres, Allen \& Unwin.

SPeCKer (Ernst), 1979, « Paul Bernays », in Boffa (Maurice), VAn Dalen (Dirk) et McAloon (Kenneth), éd., Logic colloquium '78, Proceedings of the colloquium held in Mons, August 1978, Amsterdam/Oxford, North Holland, p. 381-389.

VuILLEMIN (Jules), 1979, « La raison au regard de l'instauration et du développement scientifiques », in GERAETs (Theodore F.), éd., La Rationalité aujourd'hui, Ottawa, University of Ottawa Press, p. 67-84. 